\title{
Giant tunneling magnetoresistance with electron beam evaporated MgO barrier and $\mathrm{CoFeB}$ electrodes
}

\author{
H. Kurt, ${ }^{\text {a) }}$ K. Oguz, T. Niizeki, ${ }^{\text {b) }}$ and J. M. D. Coey \\ CRANN and School of Physics, Trinity College Dublin, Dublin 2, Ireland
}

(Received 17 December 2009; accepted 22 February 2010; published online 30 April 2010)

\begin{abstract}
Electron-beam (EB) evaporated $\mathrm{MgO}$ grows with (001) texture on amorphous $\mathrm{CoFeB}$ when the deposition rate is kept below $5 \mathrm{pm} / \mathrm{s}$. Magnetic tunnel junctions (MTJs) fabricated using this method exhibit $\sim 240 \%$ magnetoresistance at room temperature for a $2.5 \mathrm{~nm}$ thick EB-MgO barrier, which is similar to the value for a radio frequency (rf) sputtered barrier with the same junction geometry. The average barrier height of the $\mathrm{EB}-\mathrm{MgO}$ is $0.48 \mathrm{eV}$, which is higher than previously reported values for rf- $\mathrm{MgO}$ barriers and it increases with increasing annealing temperature. Our results show that EB-MgO could be a simpler alternative to rf-MgO in MTJs without any compromise in the tunnelling magnetoresistance. (C) 2010 American Institute of Physics. [doi:10.1063/1.3371811]
\end{abstract}

\section{INTRODUCTION}

Spin dependent tunnelling through a crystalline $\mathrm{MgO}$ (001) barrier has intrigued researchers worldwide due to its potential for rich physics and device applications. Following theoretical predictions, ${ }^{1,2}$ room temperature tunnelling magnetoresistance (TMR) ratios above $200 \%$ were reported in magnetic tunnel junctions (MTJs) with highly oriented $\mathrm{MgO}$ (001) tunnel barriers. ${ }^{3,4}$ Coherent crystalline growth of a bcc ferromagnet (001)/MgO (001)/bcc ferromagnet (001) MTJ structure gives rise to coherent tunnelling, which in turn leads to high TMR ratios due to the selective tunnelling of electrons with $\Delta_{1}$ symmetry through the $\mathrm{MgO}$ barrier. ${ }^{1,2}$ Such MTJs form the basis of the new generation of read head sensors, high speed nonvolatile magnetic memories such as spin torque magnetic random access memories, and spin transfer torque (STT) nano-oscillator microwave sources. ${ }^{5}$ The $\mathrm{MgO}$ (001) tunnel barriers are usually grown by radio frequency (rf) sputtering or molecular beam epitaxy (MBE) in high or ultrahigh vacuum (UHV) with very low $\mathrm{H}_{2} \mathrm{O}$ partial pressure due to hygroscopic nature of $\mathrm{MgO}$. We show here that high quality $\mathrm{MgO}$ barrier layers can also be grown by electron beam (EB) evaporation on top of amorphous $\mathrm{CoFeB}$.

Growth and postannealing optimization using borondoped $\mathrm{CoFe}$ amorphous electrodes has resulted in record TMR values in excess of $600 \%$ at room temperature in devices with no exchange bias layer and almost atomically flat interfaces, by suppression of diffusion of Ta from underlayer. ${ }^{6}$ Sufficiently smooth interfaces are crucial to get high TMR ratios and they can most easily be obtained by growing $\mathrm{MgO}$ on amorphous $\mathrm{CoFeB}$ or $\mathrm{NiFeB}$ and postannealing the MTJ stack above $350{ }^{\circ} \mathrm{C}$ to crystallize bcc $\mathrm{CoFe}$ or NiFe using the $\mathrm{MgO}$ (001) barrier as a template. ${ }^{7,8}$ During annealing, boron diffuses away to permit crystallization of bcc $\mathrm{CoFe}$ or $\mathrm{NiFe}$ through a $45^{\circ}$ in-plane rotation toward (110) direction on $\mathrm{MgO}(001)$ to form an epitaxial structure

\footnotetext{
${ }^{\text {a)} E l e c t r o n i c ~ m a i l: ~ k u r t h @ t c d . i e . ~}$

${ }^{\mathrm{b})}$ Present address: National Institute for Materials Science, 1-2-1 Sengen, Tsukuba, Ibaraki 305-0047, Japan.
}

at the interface. Achieving high TMR values also depends on the quality of the crystallinity of $\mathrm{MgO}$ as well as bcc ferromagnetic electrodes. In most MTJ stacks, the bottom $\mathrm{CoFeB}$ is pinned using a bottom pinned synthetic antiferromagnet (SAF) and a Ta layer is deposited adjacent to top CoFeB layer as a boron absorber. However, during annealing $\mathrm{Mn}$, $\mathrm{Ru}, \mathrm{Ta}$, and $\mathrm{B}$ can diffuse into $\mathrm{MgO}$ barrier, which degrades the $\mathrm{MgO}$ quality and reduces TMR. ${ }^{9}$ Recently obtained electron-energy-loss spectroscopy data have found $\mathrm{B}$ in the $\mathrm{MgO}$ barriers fabricated using B-doped amorphous ferromagnetic electrodes in addition to $\mathrm{B}$ inclusions in the adjacent Ta layers. ${ }^{10}$ Despite the significant levels of $\mathrm{B}$ diffusion in rf-MgO, high TMR values were preserved in the low resistance $\times$ area (RA) regime of MTJs.

A simpler alternative to MBE or rf sputtering is to grow high quality $\mathrm{MgO}$ (001) barriers by EB evaporation. Such EB-MgO (001) layers have been grown earlier ${ }^{11,12}$ but high TMR ratios with $\mathrm{EB}-\mathrm{MgO}$ barrier deposited on B-doped electrodes have not been demonstrated. Here, we show that MTJs fabricated using EB-MgO can exhibit 240\% TMR at room temperature, which is comparable to that of rf- $\mathrm{MgO}$ MTJs, fabricated using the same stacks.

\section{SAMPLES}

Our sample geometry consists of $\mathrm{Ta}(5) / \mathrm{Ru}(30) /$ $\mathrm{Ta}(5) / \mathrm{Ni}_{80} \mathrm{Fe}_{20}(5) / \mathrm{Ir}_{22} \mathrm{Mn}_{78}(10) / \mathrm{Co}_{90} \mathrm{Fe}_{10}(2.5) / \mathrm{Ru}(0.9) /$ $\mathrm{Co}_{40} \mathrm{Fe}_{40} \mathrm{~B}_{20}(3) / \mathrm{MgO}(t) / \mathrm{Co}_{40} \mathrm{Fe}_{40} \mathrm{~B}_{20}(3) / \mathrm{Ta}(5) / \mathrm{Ru}(10) \quad$ (all thicknesses in $\mathrm{nm}$ ) with $t=1.2,1.5,2$, and $2.5 \mathrm{~nm}$ [see inset in Fig. 1(a)]. The MTJ stacks were deposited on $100 \mathrm{~mm}$ thermally oxidized $\mathrm{Si}$ wafers in a cluster deposition system based on a Shamrock sputtering tool. All metallic layers were dc-magnetron sputtered at room temperature using $9 \mathrm{~N}$ purity argon from a heated purifier fed with $6 \mathrm{~N}$ purity argon. The bottom and top electrodes were deposited in a high vacuum (HV) chamber with a base pressure of 1 $\times 10^{-7}$ Torr. After the deposition of bottom electrodes, without breaking the vacuum, the wafer is transferred to the UHV chamber with a base pressure of $2.1 \times 10^{-10}$ Torr to deposit the active part of the MTJ stack (CoFeB/EB-MgO/CoFeB). A Telemark UHV linear e-beam source with circular beam 

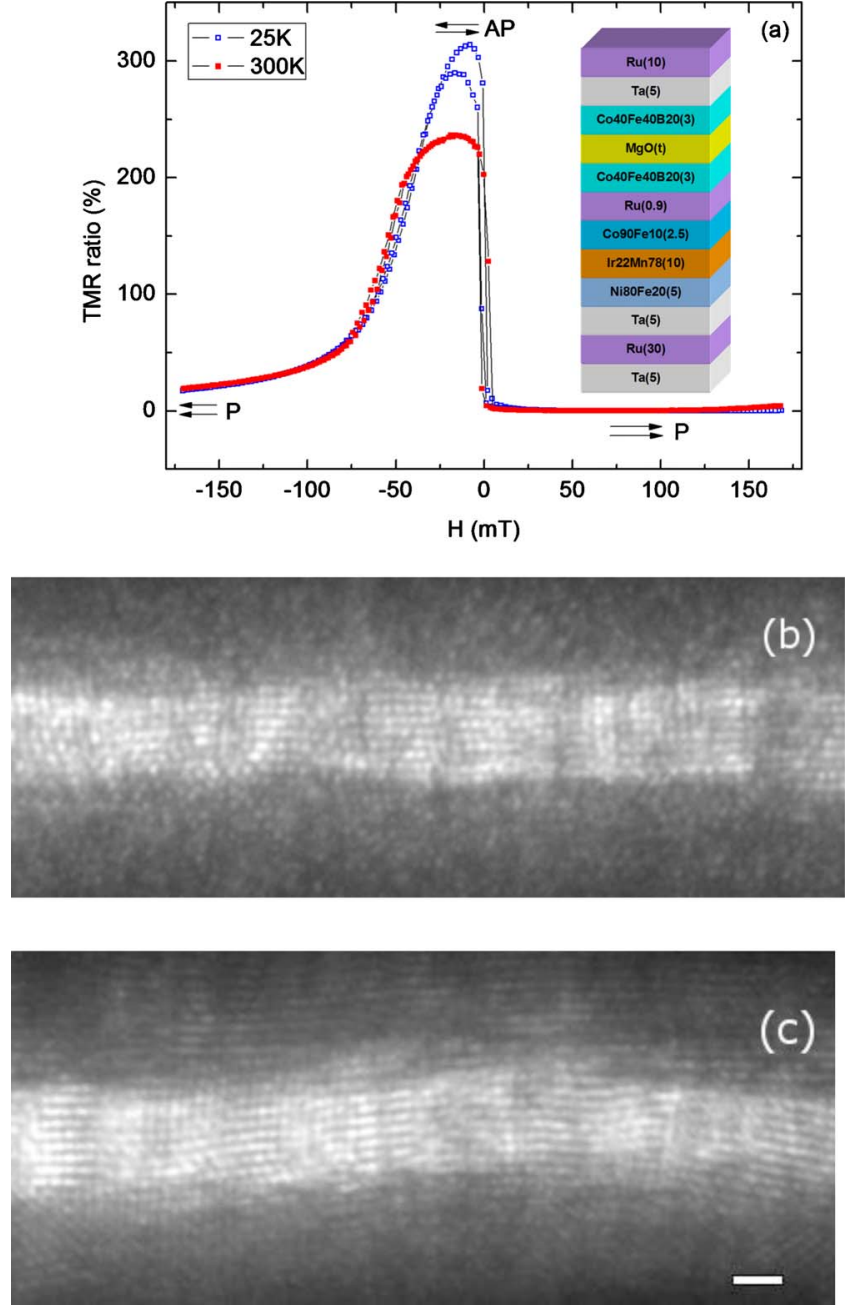

FIG. 1. (Color online) (a) TMR ratio vs magnetic field at $25 \mathrm{~K}$ (open symbols) and $300 \mathrm{~K}$ (closed symbols) for an MTJ with $2.5 \mathrm{~nm}$ e-beam $\mathrm{MgO}$ barrier, annealed at $400{ }^{\circ} \mathrm{C}$ for $1 \mathrm{~h}$. The arrows show the $\mathrm{P}$ and $\mathrm{AP}$ configurations of the magnetizations of the two $\mathrm{CoFeB}$ layers. The inset shows the MTJ stack geometry (all thicknesses in $\mathrm{nm}$ ), (b) a HR-TEM image of the as-grown EB-MgO barrier, and (c) a HR-TEM image of the EB-MgO barrier after annealing at $400{ }^{\circ} \mathrm{C}$ (scale bar is $1 \mathrm{~nm}$ ), showing the bcc crystallization of adjacent $\mathrm{CoFe}$ layers.

sweep was used to deposit $\mathrm{MgO}$. Then, the wafer is transferred back to the HV chamber to complete the stack. The active part of the rf-MgO MTJs was grown in another HV chamber of the Shamrock Cluster with a base pressure of $<1 \times 10^{-8}$ Torr, where the rf-MgO was grown using a target-facing-target sputter gun and $9 \mathrm{~N}$ purity Ar. In both cases, $4 \mathrm{~N}$ purity sintered $\mathrm{MgO}$ sources were used. A bottom synthetic antiferromagnet (SAF) is used to pin the magnetization of the bottom $\mathrm{CoFeB}$ while the magnetization of the top $\mathrm{CoFeB}$ is free to rotate.

The quality of $\mathrm{MgO}$ (001) grown on $\mathrm{CoFeB}$ using $\mathrm{EB}$ evaporation is highly dependent on the deposition rate. We have found that a rate lower than $\sim 5 \mathrm{pm} / \mathrm{s}$ is necessary to crystallize $\mathrm{MgO}$ with (001) out of plane texture at room temperature. Higher rates result in amorphous $\mathrm{MgO}$. We used $\sim 3 \mathrm{pm} / \mathrm{s}$ deposition rate for the $\mathrm{EB}-\mathrm{MgO}$ layers in the present study. The chamber pressure during $\mathrm{MgO}$ evaporation was $2-4 \times 10^{-9}$ Torr. Micron-sized junctions were fabricated using standard photolithography and argon ion mill-

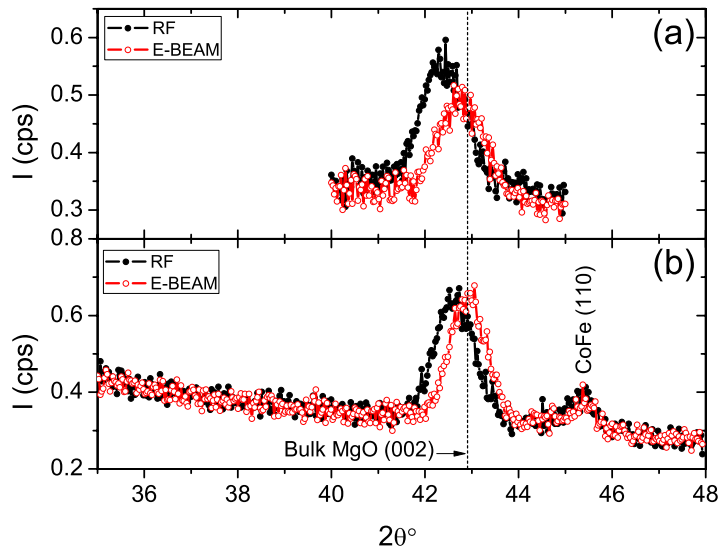

FIG. 2. (Color online) XRD data of $10 \mathrm{~nm}$ thick rf and EB-MgO sandwiched between $\mathrm{CoFeB}$ layers, (a) as grown and (b) annealed at $400{ }^{\circ} \mathrm{C}$ for $1 \mathrm{~h}$.

ing techniques. Postannealing of samples was performed in $\mathrm{HV}\left(3-4 \times 10^{-6}\right.$ Torr) in an in-plane magnetic field of $0.8 \mathrm{~T}$ for $1 \mathrm{~h}$ to crystallize CoFeB layers and to pin the bottom SAF structure. Magnetotransport measurements were made at room temperature using the conventional four-terminal method.

\section{RESULTS AND DISCUSSION}

Figure 1(a) shows typical room temperature and $25 \mathrm{~K}$ magnetoresistance curves for a $2.5 \mathrm{~nm}$ thick EB-MgO MTJ, postannealed at $400{ }^{\circ} \mathrm{C}$. TMR ratios in excess of $240 \%$ at room temperature and $312 \%$ at $25 \mathrm{~K}$ were obtained for EB$\mathrm{MgO}$ MTJs, which are comparable to those of rf-MgO MTJs. A cross-sectional high-resolution transmission electron microscopy (HR-TEM) image of an as-deposited EB-MgO MTJ is shown in Fig. 1(b) revealing the highly crystalline structure of our EB-MgO barrier. The CoFeB electrodes are found to be amorphous as expected. After annealing at $400{ }^{\circ} \mathrm{C}$, the $\mathrm{CoFeB}$ electrodes are also crystallized as seen in the transmission electron microscopy (TEM) micrograph in Fig. 1(c).

To compare the crystal structure of rf versus EB-MgO samples, we fabricated $\mathrm{CoFeB}(60) / \mathrm{MgO}(10) / \mathrm{CoFeB}(20)$ structures and postannealed them at $400{ }^{\circ} \mathrm{C}$ for $1 \mathrm{~h}$. Figure 2 shows $2 \theta \mathrm{x}$-ray diffraction (XRD) scans of these $10 \mathrm{~nm}$ thick $\mathrm{MgO}$ samples obtained using $\mathrm{Cu} K \alpha_{1}$ radiation. In the asgrown state, $\mathrm{EB}-\mathrm{MgO}$ has a d-spacing that is almost exactly the same as that of bulk $\mathrm{MgO}\left(\mathrm{a}_{0}=4.211 \AA\right)$, whereas the rf- $\mathrm{MgO}$ shows a slight increase in the d-spacing. Upon annealing, both $\mathrm{MgO}$ layers have improved crystalline structures, revealed by the identical full width at half maximum values in the $2 \theta$ scans [Fig. 2(b)]. The decrease in the $\mathrm{d}$-spacing of the rf- $\mathrm{MgO}$ is 1.07 p.m., whereas the decrease in the d-spacing of the EB-MgO is $0.92 \mathrm{pm}$ after annealing. Upon annealing, EB-MgO has the same d-spacing as bulk $\mathrm{MgO}$ but the rf- $\mathrm{MgO}$ remains at a higher d-spacing as shown in Fig. 2(b). This could be due to different characteristics of the defect and impurity distributions in both samples. To compare the initial growth of $\mathrm{EB}-\mathrm{MgO}$ with that of rf- $\mathrm{MgO}$, we attempted to grow it on amorphous thermal $\mathrm{SiO}_{\mathrm{x}}$. Our $\mathrm{EB}-\mathrm{MgO}$ would never crystallize on top of $\mathrm{SiO}_{\mathrm{x}}$ unlike rf- 


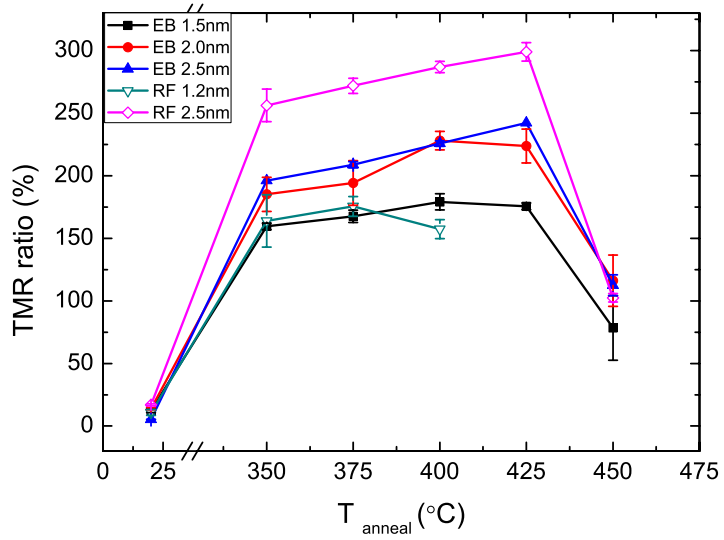

FIG. 3. (Color online) TMR ratio vs annealing temperature of EB-(closed symbols) vs rf-(open symbols) MgO MTJs.

$\mathrm{MgO}$, which is known to grow with (001) out of plane texture after forming few nanometer of amorphous $\mathrm{MgO} .{ }^{13}$ This indicates that the initial growth characteristics of EB-MgO are different from those of conventional rf-MgO.

Figure 3 summarizes the TMR ratio versus annealing temperature of both $\mathrm{EB}$ and $\mathrm{rf}-\mathrm{MgO}$ MTJs. In the as-grown state, all samples show low TMR values $(\sim 10 \%)$ due to incoherent tunneling. Upon annealing, B diffuses away and a coherent epitaxial structure, confirmed by HR-TEM, is created giving rise to coherent tunneling and high TMR values. In our samples, we observe an increase in the TMR value in both kinds of samples upto an annealing temperature of $425^{\circ} \mathrm{C}$. The highest TMR $(\sim 240 \%)$ for the $\mathrm{EB}-\mathrm{MgO}$ samples was observed for the MTJ with a $2.5 \mathrm{~nm}$ barrier. TMR values gradually decrease as the EB-MgO thickness is reduced. An MTJ with $1.2 \mathrm{~nm}$ EB-MgO barrier showed a TMR of $\sim 120 \%$ with an RA of $100 \Omega \mu \mathrm{m}^{2}$. The exchange

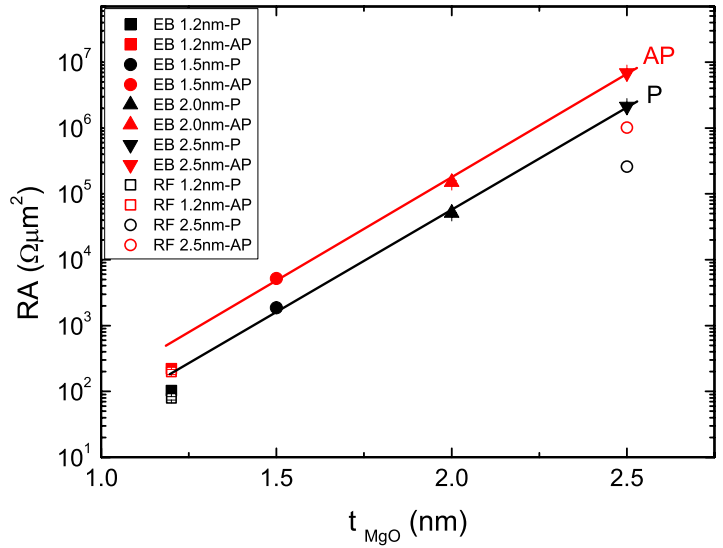

FIG. 5. (Color online) RA product vs $\mathrm{MgO}$ barrier thickness of EB-MgO MTJs (closed symbols) and rf-MgO MTJs (open symbols) in both P and AP states, annealed at $400{ }^{\circ} \mathrm{C}$ for $1 \mathrm{~h}$. The error bars are smaller than the symbols. The lines are fits to the data for $t_{\mathrm{MgO}} \geq 1.5 \mathrm{~nm}$.

bias falls dramatically on annealing above $400{ }^{\circ} \mathrm{C}$ (see Fig. 4) due to significant loss of Mn from the antiferromagnetic IrMn layer. The higher temperature stability of the antiparallel (AP) state and therefore the high TMR in our MTJs is due to the thicker Ru layer in SAF structure, which serves as a barrier for $\mathrm{Mn}$ diffusion into $\mathrm{MgO}$ barrier. ${ }^{9}$ In the case of $450{ }^{\circ} \mathrm{C}$ annealing, the exchange bias is almost annihilated and a stable AP configuration is not achieved as shown in Fig. 4(d). We believe the reason for the drop in TMR ratios for the samples annealed at this temperature is mainly due to the lack of a stable AP state.

Figure 5 shows the RA product of $\mathrm{EB}$ versus rf- $\mathrm{MgO}$ MTJs for both parallel (P) and AP configurations. Both EB$\mathrm{MgO}$ (closed symbols) and rf-MgO (open symbols) samples show an exponential increase in the RA values with increas-
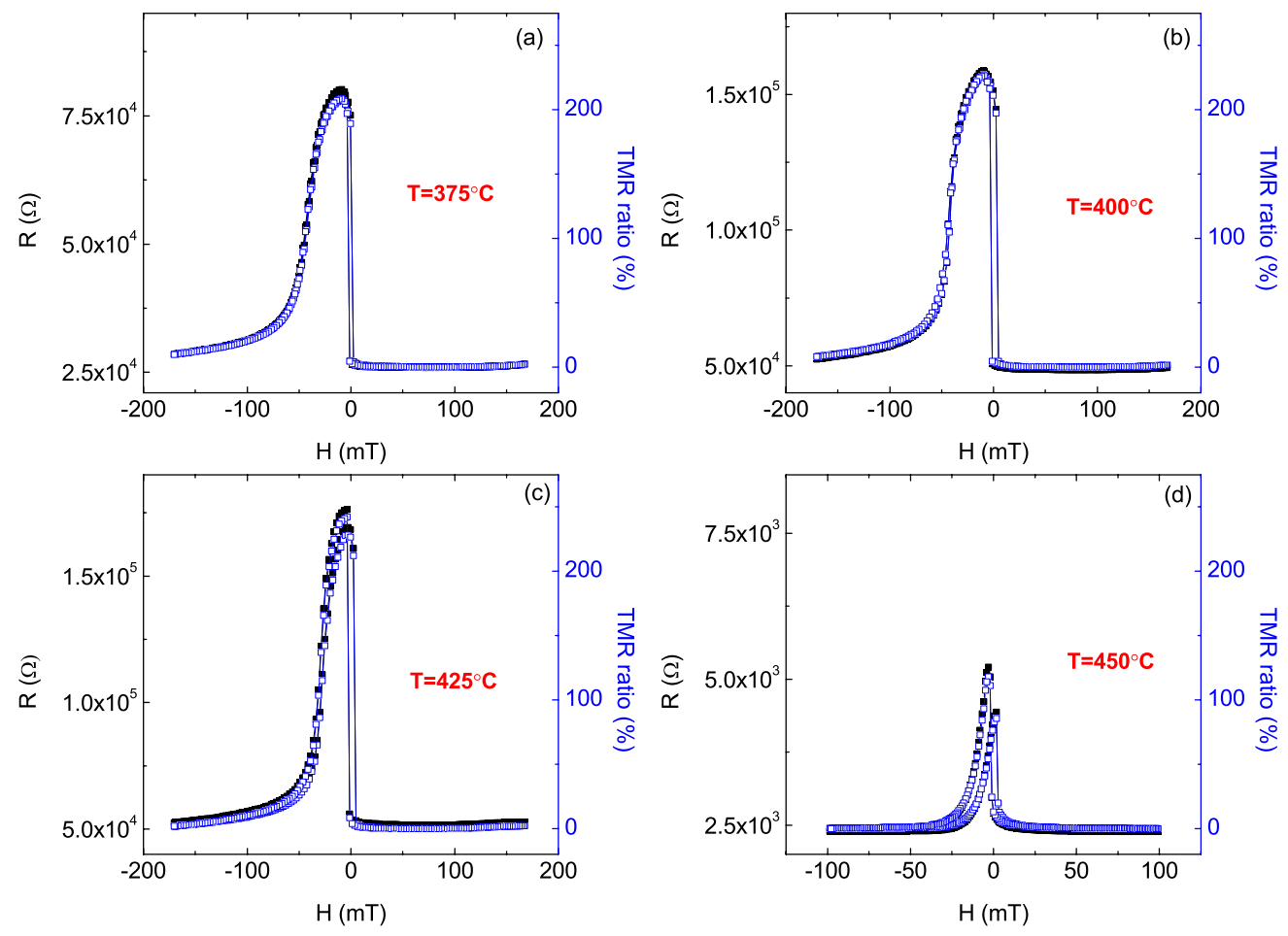

FIG. 4. (Color online) Variation in exchange bias, resistance, and TMR ratio after annealing at (a) $375^{\circ} \mathrm{C}$, (b) $400{ }^{\circ} \mathrm{C}$, (c) $425^{\circ} \mathrm{C}$, and (d) $450{ }^{\circ} \mathrm{C}$. 

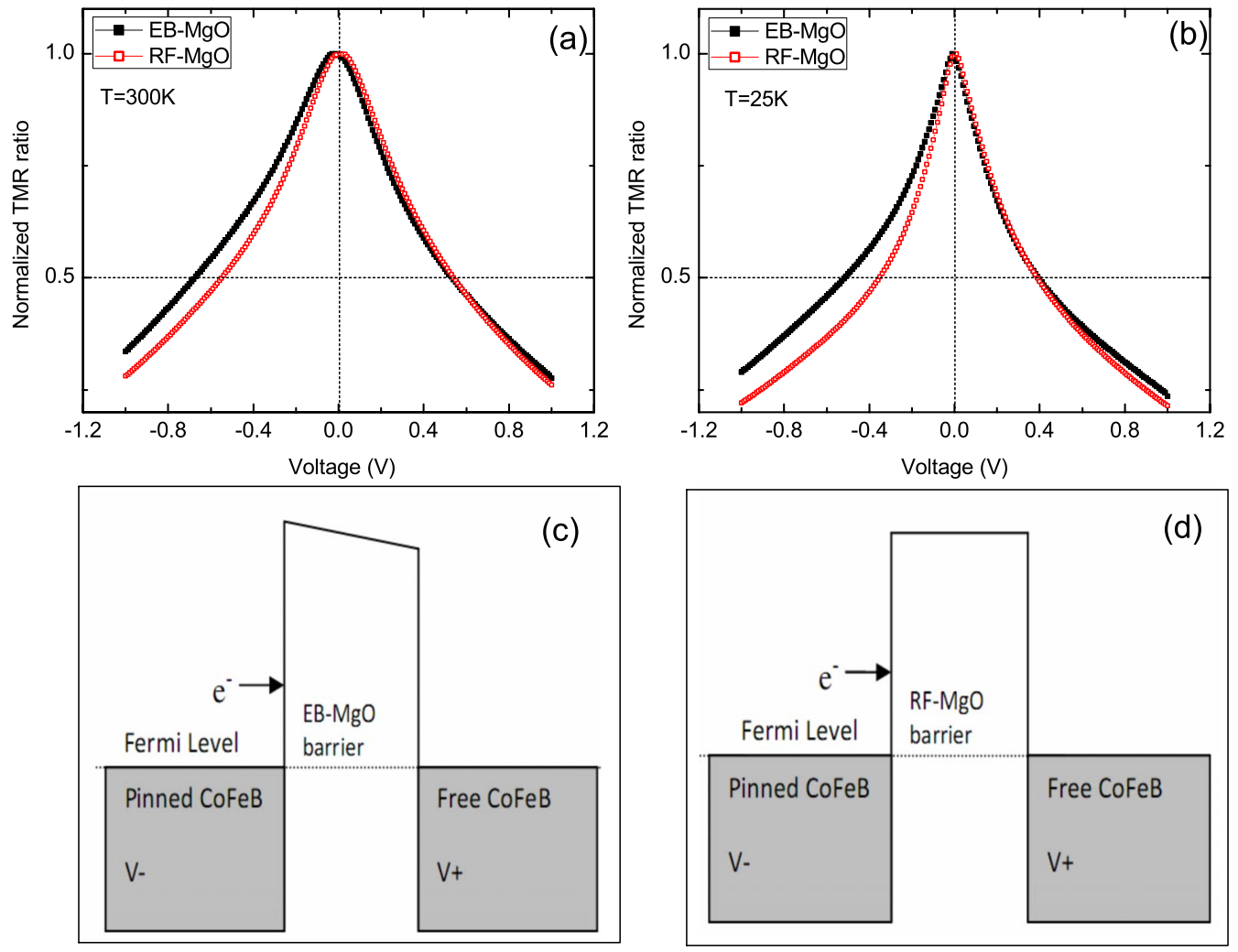

FIG. 6. (Color online) Normalized TMR ratios of rf- and EB-MgO MTJs at (a) $300 \mathrm{~K}$ and (b) $25 \mathrm{~K}$ as a function of applied bias. [(c) and (d)] Schematic of bias polarity and band diagrams of the MTJs with EB- and rf-MgO barriers, respectively.

ing $\mathrm{MgO}$ thickness. Straight lines are drawn as guides to the eye to show the exponential increase. The deviations from this line in the low RA regime are common due to the highly pronounced effect of the interfacial roughness for thin barriers. Barrier heights of $0.47 \mathrm{eV}$ for $\mathrm{P}$ and $0.49 \mathrm{eV}$ for $\mathrm{AP}$ states were calculated from linear fits to the data points for $t_{\mathrm{MgO}} \geq 1.5 \mathrm{~nm}$ using Wenzel-Kramer-Brillouin (WKB) approximation in the low voltage limit. ${ }^{14}$ These values are higher than previously reported values for the rf- $\mathrm{MgO}(0.34$ $0.38 \mathrm{eV})^{15}$ and MBE grown $\mathrm{MgO}(0.39 \mathrm{eV})^{4}$ barriers, but much smaller than that for an ideal $\mathrm{MgO}$ tunnel barrier (3.7 eV). ${ }^{16}$ Significant deviations in the barrier heights of the $\mathrm{MgO}$ in MTJs from that of an ideal $\mathrm{MgO}$ could be attributed to the oxygen vacancies in the grown barriers. ${ }^{4}$ The greater barrier height of the $\mathrm{EB}-\mathrm{MgO}$ barrier is most likely due to the lower defect densities compared to the rf- $\mathrm{MgO}$, which reveals itself in the $2 \theta$ XRD scans as shown in Fig. 2. A greater barrier height could be advantageous for sensor and STT applications, where a higher voltage output is required. In addition, the $\mathrm{EB}-\mathrm{MgO}$ barrier may exhibit lower noise levels on account of the low defect density.

The relevant TMR value for STT switching is the value at the switching current (voltage) which is typically about half of the maximum TMR. We determined the bias dependences of our samples using the I-V plots in P and AP states and they are plotted in Fig. 6. The rf-MgO MTJ shows an almost symmetric decrease of TMR as a function of bias but the EB-MgO MTJ shows an asymmetric behavior. For negative bias (electrons tunneling from free to pinned layer), the TMR of the EB-MgO sample decreases at a lower rate than that for the positive bias, whereas the rate of drop in TMR of rf- $\mathrm{MgO}$ sample as a function of bias is faster but almost same for both polarities. For electrons tunnelling from the free layer to the pinned layer, the tunnelling probability is lower at the same bias, therefore the negative bias needs more energy for field emission tunnelling, which is due to the asymmetric nature of top and bottom interfaces of the EB-MgO. This asymmetry could be explained by the differences in the initial growth characteristics of rf versus EB$\mathrm{MgO}$. In rf sputtering, the sputtered species are more energetic than those for the EB evaporated ones. Our attempts to grow $\mathrm{EB}-\mathrm{MgO}$ on thermal $\mathrm{SiO}_{\mathrm{x}}$ did not result in a crystalline $\mathrm{MgO}$, whereas it is known that the rf- $\mathrm{MgO}$ can be grown with (001) out of plane texture on $\mathrm{SiO}_{\mathrm{x}}$. The top $\mathrm{MgO} /$ $\mathrm{CoFeB}$ interfaces are almost the same for both samples; the I-V curves show similar bias dependence at positive voltage. The same asymmetric bias dependence of TMR is also observed at $25 \mathrm{~K}$ for the $\mathrm{EB}-\mathrm{MgO}$ sample, however, the rate of drop in TMR is higher at low temperatures.

Differential conductance measurements of both types of samples display different behavior for coherent transport in the P state as shown in Figs. 7(a) and 7(c). For electrons tunnelling from free to pinned layer (negative bias), the differential conductance minimum is higher than those for electrons tunnelling in the reverse direction for the EB-MgO MTJ, and vice-versa for the rf-MgO MTJ. The asymmetry of coherent tunneling at the interfaces in the $\mathrm{P}$ state is more pronounced in the rf-MgO MTJ sample, which corroborates the argument about the different initial growth characteristics for different $\mathrm{MgO}$ growth methods. 

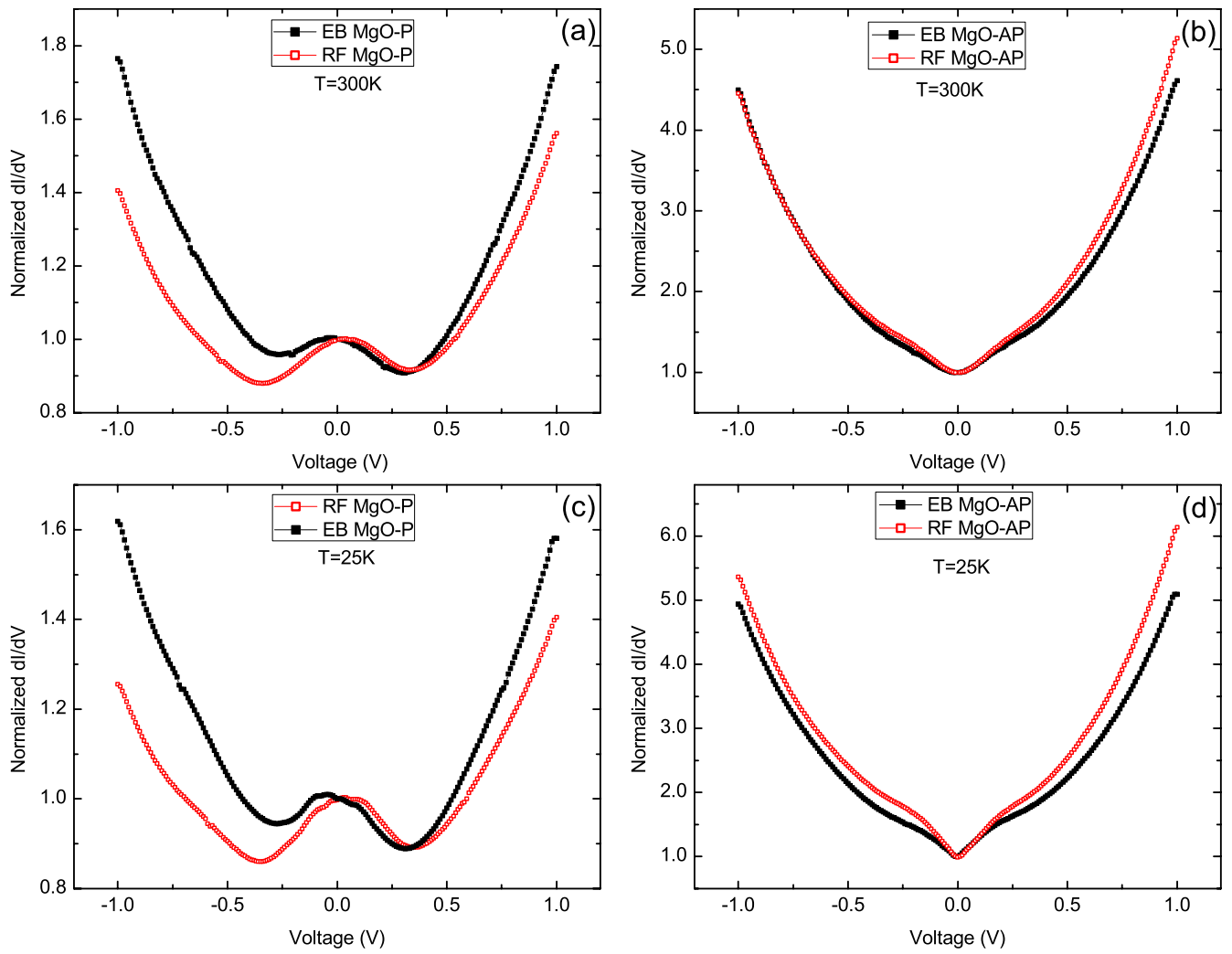

FIG. 7. (Color online) Normalized differential conductance vs bias graphs for rf-MgO MTJs (open symbols) vs EB-MgO MTJs (closed symbols) postannealed at $400{ }^{\circ} \mathrm{C}$ for a barrier thickness of $2.5 \mathrm{~nm}$ in $\mathrm{P}$ and $\mathrm{AP}$ states at 300 and $25 \mathrm{~K}$, respectively.

Figure 8 shows the variation in resistance with annealing temperature of both EB- and rf- $\mathrm{MgO}$ samples upto $425{ }^{\circ} \mathrm{C}$. For the rf-MgO samples the resistance has a tendency to decrease with increasing annealing temperature, whereas for the EB-MgO samples the resistance has a tendency to increase in both $\mathrm{P}$ and AP states. This is probably due to structural differences in these $\mathrm{MgO}$ barriers. The increase in the resistances of $\mathrm{EB}-\mathrm{MgO}$ samples indicates that the barrier height increases with increasing annealing temperature. However, for the rf- $\mathrm{MgO}$ case, as the annealing temperature is increased, the insulating property of the rf- $\mathrm{MgO}$ deteriorates despite improved crystallinity, which may be attributed

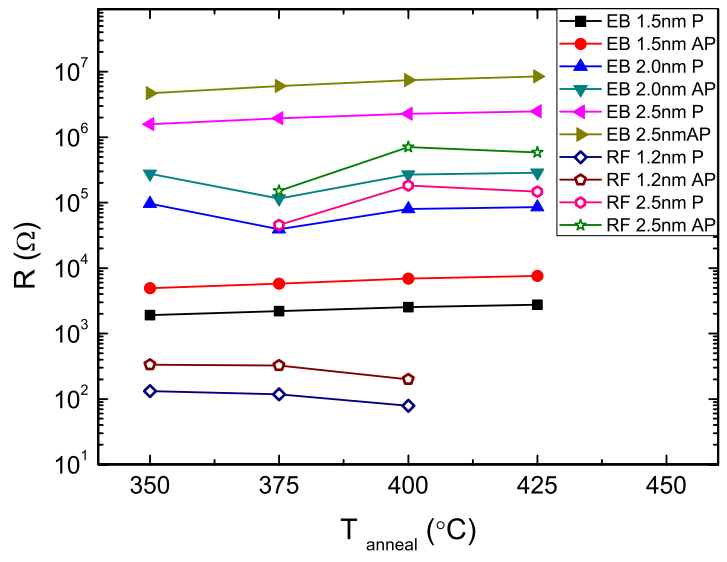

FIG. 8. (Color online) Resistance vs annealing temperature for 4 $\times 12 \mu \mathrm{m}^{2}$ junctions in $\mathrm{P}$ and AP states at $300 \mathrm{~K}$. Error bars are smaller than the symbols. to increasing levels of impurities, such as B, Mn, or Ta diffusing into the rf- $\mathrm{MgO}$ barrier. This shows $\mathrm{EB}-\mathrm{MgO}$ is more resistant to diffusion during postannealing.

\section{CONCLUSIONS}

We have achieved TMR ratios in excess of $240 \%$ at room temperature and $312 \%$ at $25 \mathrm{~K}$ using $\mathrm{EB}-\mathrm{MgO}$ barriers grown on amorphous $\mathrm{CoFeB}$ electrodes. The (001) texture is obtained by controlling the $\mathrm{MgO}$ deposition rate below 5 $\mathrm{pm} / \mathrm{s}$. The average calculated barrier height of our EB-MgO is higher than those for previously reported values of rf- $\mathrm{MgO}$ with similar TMR ratios, which is thought to be due to a lower defect density in the $\mathrm{EB}-\mathrm{MgO}$ barrier. This could be advantageous for STT oscillator applications that require high voltage outputs as well as for sensor applications which require a lower low-frequency noise. The asymmetric behavior of the TMR drop as a function of bias for the EB-MgO reveals the asymmetry of the top and bottom interfaces, whereas the rf- $\mathrm{MgO}$ barrier has a more symmetric structure.

Structural differences in the $\mathrm{MgO}$ layers are revealed by $2 \theta$ scans performed on the thick $\mathrm{EB}$ and $\mathrm{rf}-\mathrm{MgO}$. It was found that the $\mathrm{EB}-\mathrm{MgO}$ has a d-spacing that matches that of bulk $\mathrm{MgO}$, whereas the rf- $\mathrm{MgO}$ shows a slight increase in the d-spacing, which could be attributed to a higher density of oxygen vacancies created during rf sputtering. Further studies are needed to understand the cause of the differences in the d-spacings of both samples. Increases in the resistance 
of EB samples and the decreases in resistance of rf samples with increasing annealing temperature could be due to a more diffusion resistant nature of $\mathrm{EB}-\mathrm{MgO}$.

Regardless of differences in their structures, initial growth, and annealing temperature behaviors, the TMR ratios achieved using EB-MgO barriers are comparable to those of our rf-MgO MTJs as well as those of several other groups. EB-MgO presents a simpler alternative to rf- $\mathrm{MgO}$, which can be implemented at the wafer scale.

\section{ACKNOWLEDGMENTS}

The authors thank Karsten Rode for X-ray measurements and discussions, Colm Faulkner and Markus Boese for the HR-TEM images. This research was supported by Science Foundation Ireland (SFI) as part of the MANSE project (contract no. SFI 05/IN/1850).

${ }^{1}$ W. H. Butler, X. G. Zhang, T. C. Schulthess, and J. M. MacLaren, Phys. Rev. B 63, 054416 (2001).

${ }^{2}$ J. Mathon and A. Umerski, Phys. Rev. B 63, 220403(R) (2001).

${ }^{3}$ S. S. P. Parkin, C. Kaiser, A. Panchula, P. M. Rice, B. Hughes, M. Samant, and S.-H. Yang, Nature Mater. 3, 862 (2004).
${ }^{4}$ S. Yuasa, T. Nagahama, A. Fukushima, Y. Suzuki, and K. Ando, Nature Mater. 3, 868 (2004)

${ }^{5}$ A. M. Deac, A. Fukushima, H. Kubota, H. Maehara, Y. Suzuki, S. Yuasa, Y. Nagamine, K. Tsunekawa, D. D. Djayaprawira, and N. Watanabe, Nat. Phys. 4, 803 (2008).

${ }^{6}$ S. Ikeda, J. Hayakawa, Y. Ashizawa, Y. M. Lee, K. Miura, H. Hasegawa, M. Tsunoda, F. Matsukura, and H. Ohno, Appl. Phys. Lett. 93, 082508 (2008)

${ }^{7}$ D. D. Djayaprawira, K. Tsunekawa, M. Nagai, H. Maehara, S. Yamagata, N. Watanabe, S. Yuasa, Y. Suzuki, and K. Ando, Appl. Phys. Lett. 86, 092502 (2005)

${ }^{8}$ J. C. Read, J. J. Cha, W. F. Egelhoff, H. W. Tseng, P. Y. Huang, Y. Li, D. A. Muller, and R. A. Buhrman, Appl. Phys. Lett. 94, 112504 (2009).

${ }^{9}$ Y. M. Lee, J. Hayakawa, S. Ikeda, F. Matsukura, and H. Ohno, Appl. Phys. Lett. 89, 042506 (2006).

${ }^{10}$ J. J. Cha, J. C. Read, W. F. Egelhoff, P. Y. Huang, H. W. Tseng, Y. Li, R. A. Buhrman, and D. A. Muller, Appl. Phys. Lett. 95, 032506 (2009).

${ }^{11}$ J. J. Cha, J. C. Read, R. A. Buhrman, and D. A. Muller, Appl. Phys. Lett. 91, 062516 (2007).

${ }^{12}$ T. Taira, T. Ishikawa, N. Itabashi, K.-I. Matsuda, T. Uemura, and M. Yamamoto, J. Phys. D 42, 084015 (2009).

${ }^{13}$ S. Yuasa and D. D. Djayaprawira, J. Phys. D 40, R337 (2007).

${ }^{14}$ J. G. Simmons, J. Appl. Phys. 34, 1793 (1963).

${ }^{15}$ J. Hayakawa, S. Ikeda, F. Matsukura, H. Takahashi, and H. Ohno, Jpn. J. Appl. Phys., Part 2 44, L587 (2005).

${ }^{16}$ W. Wulfhekel, M. Klaua, D. Ullmann, F. Zavaliche, J. Kirschner, R. Urban, T. Monchesky, and B. Heinrich, Appl. Phys. Lett. 78, 509 (2001). 